\title{
Wireless Power Transfer Technology in On-Line Electric Vehicle
}

\author{
Seungyoung $\mathrm{Ahn}^{1} \cdot$ Yangbae $\mathrm{Chun}^{2} \cdot$ Dong-Ho $\mathrm{Cho}^{1} \cdot{\text { Joungho } \mathrm{Kim}^{1}}^{1}$
}

\begin{abstract}
The On-line Electric Vehicle (OLEV) is an electric transport system in which the vehicle's power is transferred wirelessly from power lines underneath the surface of the road. Advantages of the OLEV include reducing battery size and cost to about 20 percent of that of conventional battery-powered electric vehicles, thereby minimizing the vehicle's weight and price, as well as the cost of charging the system. In this paper, we introduce a wireless power transfer mechanism to maximize the electrical performance of the power transfer system. Power transfer capacity, power transfer efficiency, and magnitude of leakage in the electromagnetic field (EMF) are analyzed, and the optimization methodology of the design parameters is discussed.
\end{abstract}

Key words: Wireless Power Transfer, On-Line Electric Vehicle, Electromagnetic Field, Shielding, Optimization.

\section{Introduction}

Since Nikola Tesla's study of wireless power transfer in the beginning of the 20th century [1], there have been challenges in achieving genuine mobility in electronic devices and machines. Research on wireless power transfer technology by Soljačić brought more attention to wireless power transfer systems using magnetic resonances [2]. However, obstacles still remain to the commercialization of wireless power transfer technology, including limitations in power transfer capacity, efficiency, reliability, and safety. In order to deliver wireless power transfer technology to the market, more effort must be made to improve electrical performance, analyze standardization, and develop infrastructures for each application [3].

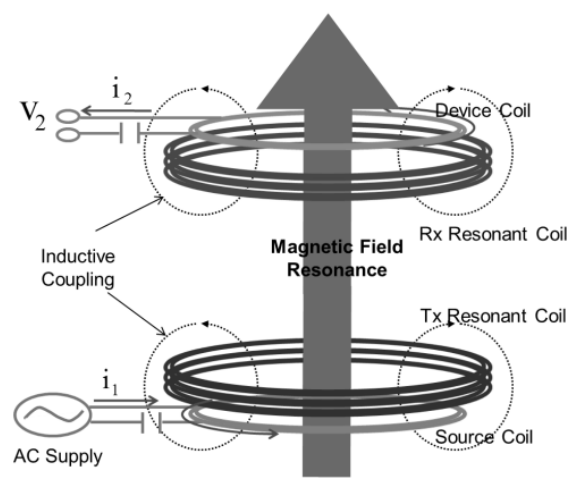

Fig. 1. Schematic of wireless power transfer systems.
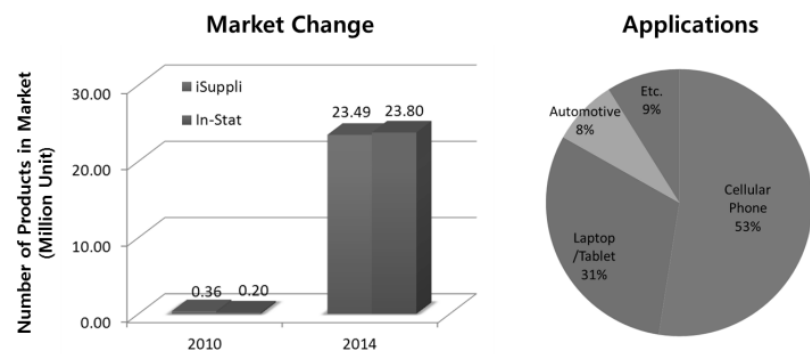

Fig. 2. Market trend of wireless power transfer system.

The application of wireless power transfer systems began with small, portable electronic devices, including mobile phones and laptop computers. However, the technology is now being transferred to the automobile market. In the field of transportation, the use of wireless power transfer systems is particularly meaningful in the realization of electric vehicles [4], [5].

Even though intensive research has been performed on fully electric transportation systems, we are still facing serious problems in battery-powered electric delivery systems. These issues include the large size, weight, and cost of batteries; long recharging times, and limited availability of charging service points. Moreover, diminished stocks of lithium could lead to increasingly high prices and ultimately price electric vehicles out of the automotive market. In light of these difficulties, the use of wireless power transfer systems in electric vehicles is recommended [6].

Manuscript received September 5, 2011 ; revised September 19, 2011. (ID No. 20110905-7J)

${ }^{1}$ Department of Electrical Engineering, Korea Advanced Institute of Science and Technology, Daejeon, Korea.

${ }^{2}$ On-Line Electric Vehicle Project, Korea Advanced Institute of Science and Technology, Daejeon, Korea.

Corresponding Author : Seungyoung Ahn (e-mail : sahn@kaist.ac.kr) 


\section{II . On-Line Electric Vehicle}

KAIST has introduced a novel on-line electric vehicle (OLEV), in which the vehicle constantly recharges via power lines beneath the surface of the road (Fig. 3). The OLEV has a minimal battery capacity (about $20 \%$ compared to that of conventional battery-powered electric vehicles), which minimizes the weight and price of both the vehicle and the power station. The design of the power lines and pick-up module determines the performance of the power transfer system and leakage in the electromagnetic field [7].

One of the key design requirements is suppression of the leakage of magnetic flux from power lines and the pick-up module in order to maintain power delivery efficiency and meet the OLEV's total power needs. In this paper, we introduce the wireless power transfer technology applied to OLEVs, and show the techniques for improving electrical performance. Optimization methodology of the design parameters and the shielding of the electromagnetic field are analyzed, and simulation and measurements for the verification are discussed.

The power transfer system for an OLEV consists of an inverter, power lines, a pick-up module, capacitors, a battery and a motor, as shown in Fig. 3. A $60 \mathrm{~Hz}$ current for power transfer is converted to $20 \mathrm{kHz}$ at the inverter stage, and a current of about 200 A flows through the power lines. The design of the power lines and pick-up module determines the electrical performance of the power transfer system.

The magnetic flux generated from the power lines is gathered at the pick-up module to generate DC power for the vehicle's motor. The non-contact power transfer that occurs between the power lines and the pick-up module generates a huge magnetic flux. Therefore, the design of the power lines and the pick-up module are the key technologies for effective power transfer and solving the electromagnetic field (EMF) problems.

Fig. 5 shows the vertical magnetic flux of the power lines and pick-up module. There are two power lines

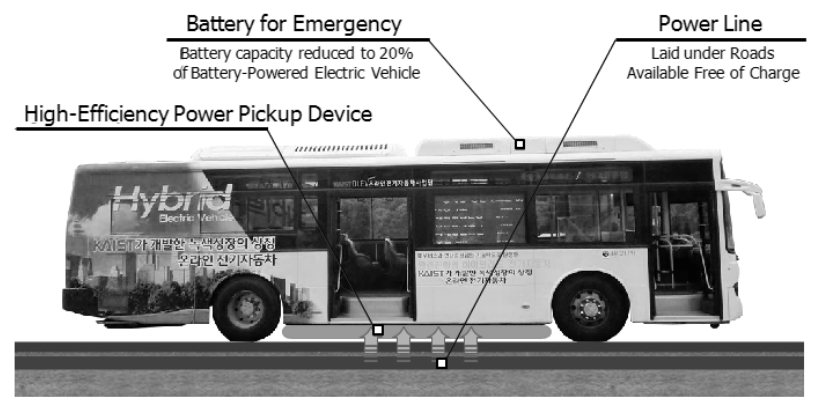

Fig. 3. Photograph of on-line electric vehicle system.

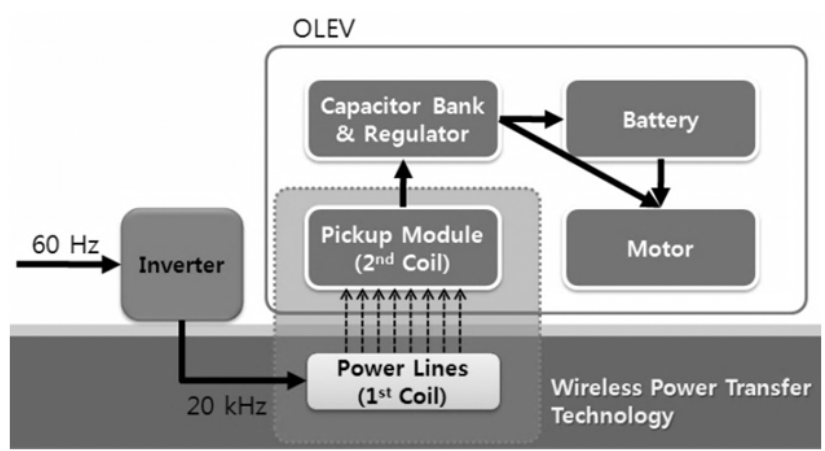

Fig. 4. Block diagram of overall system for OLEV.

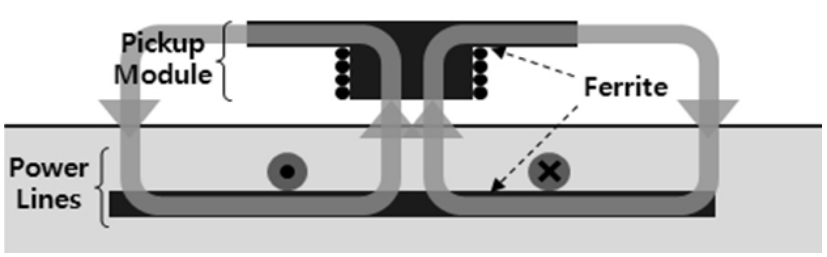

(a) Cross-sectional view

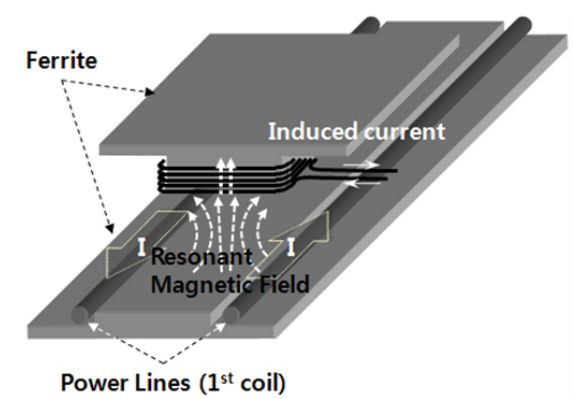

(b) Perspective view

Fig. 5. Concept of power lines and pickup coils pair and the generated magnetic flux for vertical magnetic flux type system.

with opposite current directions beneath the road surface forming a current loop. Due to the current in the power lines, a magnetic flux is induced around each power line. Between the power lines, the magnetic fluxes from the two power lines are combined. The pick-up module catches the vertical magnetic flux through copper coils around the ferrite core. This type has the advantage of providing efficient power transfer because the direction of the magnetic flux from the power lines is the same as the direction of the flux to the pick-up module.

\section{Procedure of OLEV System Design}

In designing the power lines and pick-up module structure for the OLEV system, we considered three criteria for the electrical performance of the wireless power transfer system: power transfer capability, power transfer efficiency, and leakage from the electromagnetic field. 


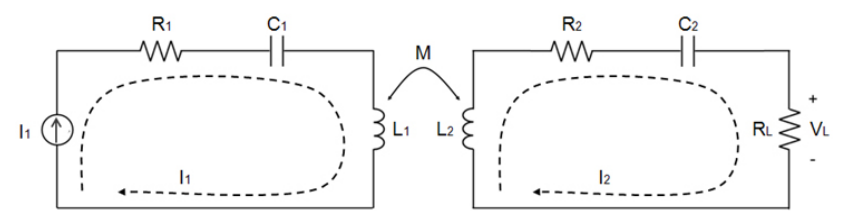

Fig. 6. Equivalent circuit model of power transfer system.

$$
\begin{gathered}
P_{L} \cong \frac{\omega^{2} M^{2}}{\left(R_{2}+R_{L}\right)^{2}+\left(\omega L_{2}-\frac{1}{\omega C_{2}}\right)^{2}} I_{1}^{2} R_{L} \cong \frac{\omega^{2} M^{2}}{R_{L}} I_{1}{ }^{2} \\
K \cong \frac{\omega^{2} M^{2} R_{L}}{R_{1}\left(R_{2}+R_{L}\right)^{2}+\omega^{2} M^{2}\left(R_{2}+R_{L}\right)} \cong \frac{1}{1+\frac{R_{1} R_{L}}{\omega^{2} M^{2}}}
\end{gathered}
$$

Power transfer capability implies the maximum power that can be transferred from the power lines under the road to the load in the vehicle, which consequently determines the vehicle's maximum speed and battery recharging time. From the simplified equivalent circuit model of the wireless power transfer system with two series resonant coils as shown in Fig. 6, the power at the load $R_{L}$ is calculated to be proportional to the frequency, mutual inductance, and magnitude of source current, assuming that the system is operating at the resonance frequency as shown in Fig. 1.

Power transfer efficiency is also an important factor for commercialization and should be reasonably high compared with other types of vehicles. To increase the efficiency, we need to minimize the loss at each stage of the OLEV power system. With the development of power components operating at $20 \mathrm{kHz}$, which were not available a few decades ago, the efficiency of the inverter has been significantly increased. Also, the mutual inductance should be increased, and the parasitic resistance $R_{1}$ and $R_{2}$ - the loss from these resistances should be decreased as shown in Fig. 6 to further increase efficiency.

The third criterion, EMF leakage, is proportional to the magnitude of the current and inversely proportional to the distance between current position and measurement position without a shield. However, as the application of passive and active shields significantly changes the magnitude of EMF, the design of the EMF should be performed separately. This will be discussed in the next section.

The previous design procedure for OLEV wireless power transfer systems is shown in Fig. 7(a). At the early stage of design, we have to determine the topology and outline of the dimensions for the physical structures, such as the number of coils, coil size and dimension, and the position of the ferrite core. This is because the mutual inductance is roughly determined when the physical dimension is fixed and it is hard to change the value significantly at a later stage. Design freedom is therefore limited and the final design parameter is difficult to optimize. For optimal design, several parameters should be considered simultaneously during the design stage. The optimization stage is necessary after the design parameters and performance parameters have been defined, as shown in Fig. 7(b). This will be discussed in section V.

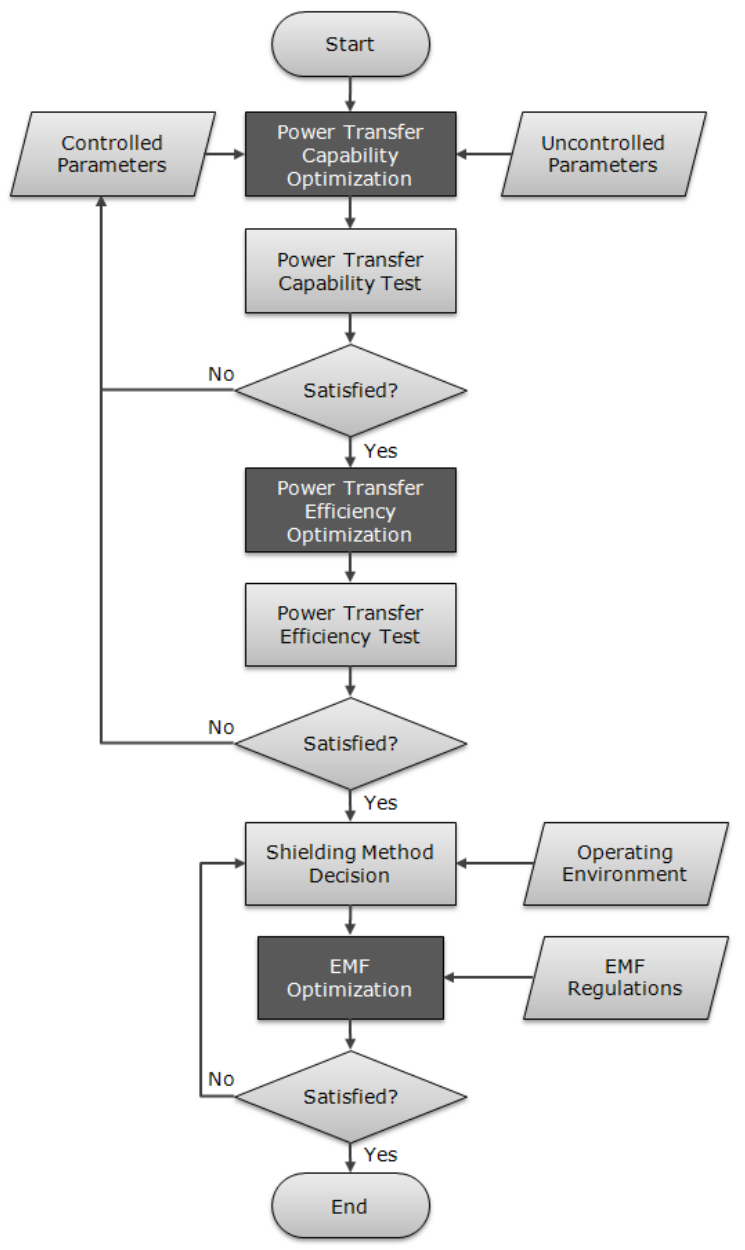

(a) Previous procedure

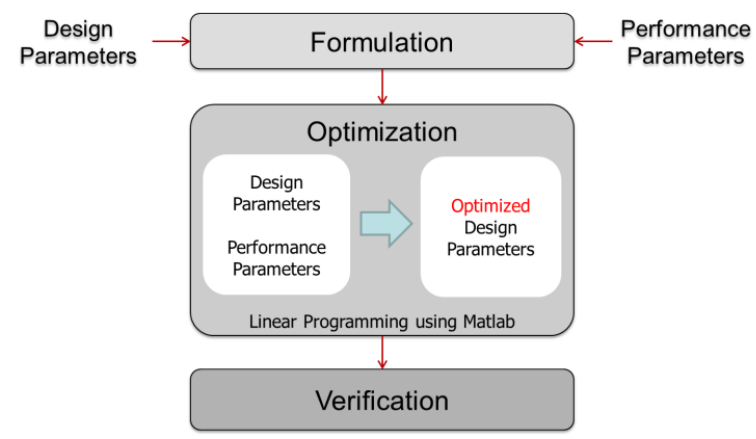

(b) Suggested procedure

Fig. 7. Design procedures of wireless power transfer system in OLEV. 
Table 1. Sensitivity analysis of transferred power for the change of design parameters.

\begin{tabular}{|l|l|c|c|c|c|}
\hline \multirow{2}{*}{ Design parameters } & \multicolumn{3}{|c|}{ Change of parameters (\%) } \\
\cline { 3 - 6 } & Air gap & -20 & -10 & +10 & +20 \\
\hline \multirow{4}{*}{$\begin{array}{l}\text { Dimension } \\
\text { parameters }\end{array}$} & $\begin{array}{l}\text { Number of turns } \\
\text { in pickup coil }\end{array}$ & -44.0 & -21.0 & +21.0 & +44.0 \\
\cline { 2 - 6 } & $\begin{array}{l}\text { Dist. between } \\
\text { rail wires }\end{array}$ & -40.0 & -18.6 & +17.1 & +35.1 \\
\cline { 2 - 6 } & $\begin{array}{l}\text { Pickup coil } \\
\text { width }\end{array}$ & -24.2 & -9.5 & +6.9 & +12.2 \\
\hline \multirow{4}{*}{$\begin{array}{l}\text { Material } \\
\text { parameters }\end{array}$} & Permeability $(\mu)$ & -1.0 & -0.4 & +0.4 & +0.72 \\
\cline { 2 - 6 } & Permittivity $(\varepsilon)$ & 0 & 0 & 0 & 0 \\
\cline { 2 - 6 } & Conductance $(\sigma)$ & 0 & 0 & 0 & 0 \\
\cline { 2 - 6 } & Frequency & -44.1 & -20.7 & +21.3 & +45.2 \\
\hline \multirow{2}{*}{$\begin{array}{l}\text { Electrical } \\
\text { parameters }\end{array}$} & Current & -44.0 & -21.0 & +21.0 & +44.0 \\
\cline { 2 - 6 } & Frequency & -44.1 & -20.7 & +21.3 & +45.2 \\
\hline
\end{tabular}

Table 1 shows the result of simulated sensitivity analysis of transferred power for the change of main design parameters, which is the reference for the optimization of the design. At each design stage, a sensitivity analysis on the effect of each design parameter has been performed using simulation with 3-dimensional field solver.

\section{Electromagnetic Field Shielding}

Fig. 8 shows the magnetic flux density distribution of OLEV. In the case of the vertical magnetic flux type, there is one magnetic flux path between the power lines and pick-up module where the power is transferred. The return flux comes back to the power lines via the sides of the main flux path. The horizontal magnetic flux type has two magnetic flux paths. The side power lines of this type have return flux paths on the side of the main flux path. The return flux path creates the fringing magnetic flux, and this flux is measured as the EMF level of OLEV. In this work, the target EMF level is $62.5 \mathrm{mG}$, according to Korea Communications Commission regu-

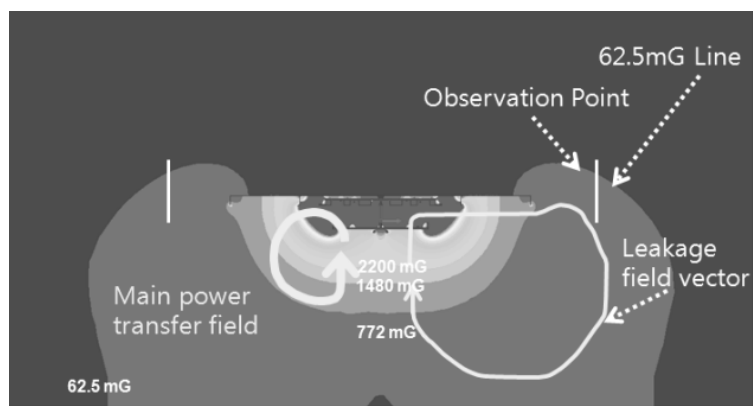

Fig. 8. Distribution of magnetic field for OLEV.

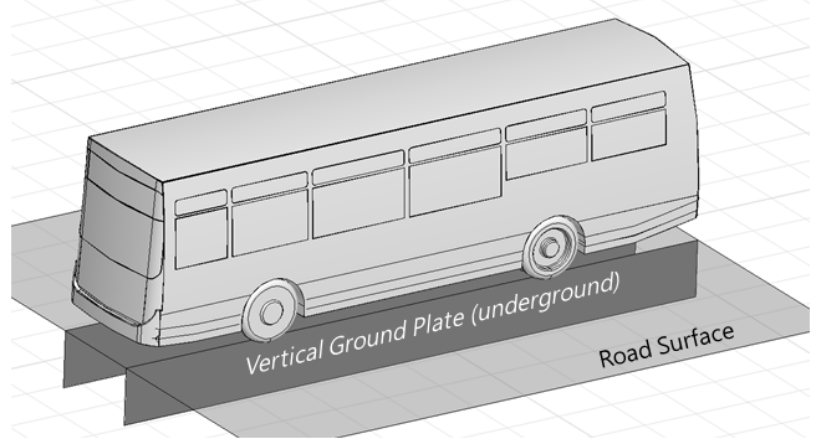

Fig. 9. Construction vertical ground plate for passive shield.

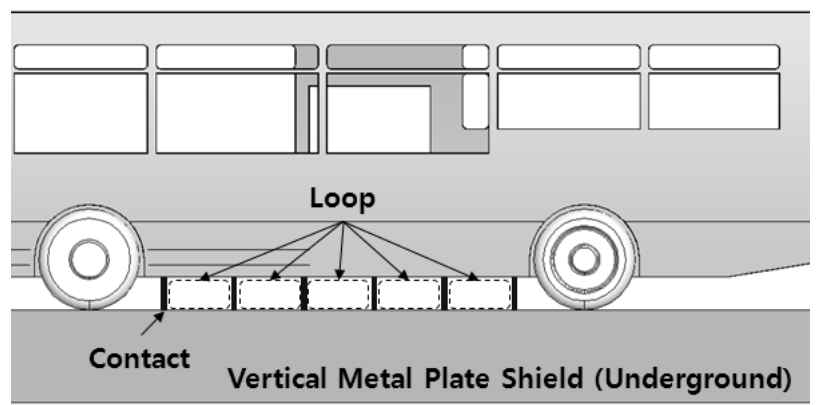

Fig. 10. Connections between vehicle body and underground vertical metal plate for passive shield.

lations, which follows the ICNIRP design guideline [8].

As the OLEV power supply system generates large amounts of magnetic field in order to transfer the 60 $\mathrm{kW}$ of power necessary for the vehicle, there are tens of thousands of $\mathrm{mG}$ of magnetic flux between the power lines and pick-up module beneath the vehicle while power is being transferred. As a result, if just $0.1 \%$ of magnetic field leakage occurs, the EMF level could exceed the regulation $62.5 \mathrm{mG}$. The distribution of magnetic field for OLEV is shown in Fig. 8.

Basically, passive shielding using metal plates is applied to OLEV in order to reduce the electromagnetic field. To protect passengers from the magnetic field, a metal plate is applied to the bottom of the vehicle. As the power lines are the source of the magnetic field, vertical plate shields are applied as shown in Fig. 9 [9], [10].

To improve effectiveness of the passive shield, we additionally applied soft contacts between the bottom plate and vertical ground plate by metal brushes, as shown in Fig. 10. The metal brush is a bundle of thin metal wires attached beneath the bottom plate which connect the current path between the vehicle body and the ground plate beneath the road surface. A photograph of the implemented metal brush is shown in Fig. 11. The number 


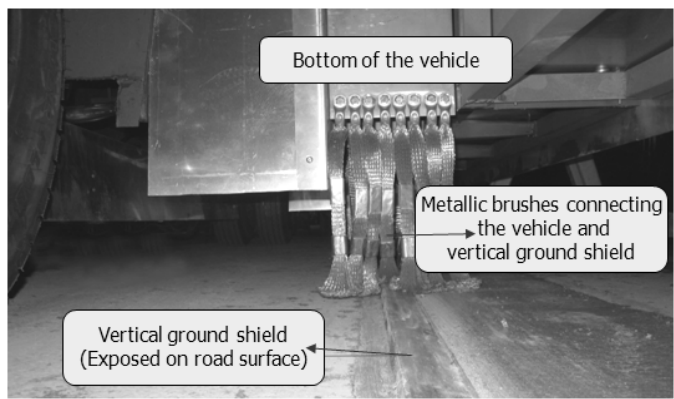

Fig. 11. Photo of implemented metal brush in passive shield.

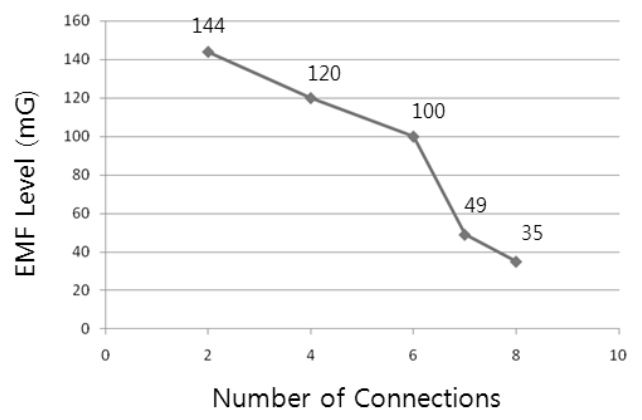

Fig. 12. Effect of the number of connections between the metallic vehicle body and the horizontal ground shield.

of connections using metal brushes is a significant factor in improving the effectiveness of the passive shielding. The EMF level decreases from $144 \mathrm{mG}$ to $35 \mathrm{mG}$ when the number of connections using metal brushes is increased from 2 to 8, as shown in Fig. 12.

The EMF can be minimized by active shielding with or without passive shields independently, and the basic concept of the active shield is shown in Fig. 13. Similar to power lines, the active shield is also a metal wire which carries the same frequency with current, but the phase is the opposite of the current in the pick-up [11].

In the design of the active shield, the directions of magnetic fields by the source and the active shield should be carefully considered. In Fig. 14, the direction of magnetic field is shown. To make the EMF level less than the regulation at all positions, the magnetic field from the active shield should be almost the same as that from the pick-up module at all positions. More than 20 $\mathrm{cm}$ above the road surface, the magnetic field vector is parallel to the metal plate because of the metallic shield at the bottom of the vehicle. Therefore, placing the active shield close to the pick-up coil is more effective. However, if the active shield is closer to the pick-up coil, the current of the active shield should be larger. For this reason, the placement of the active shield is compromised considering the shielding effectiveness

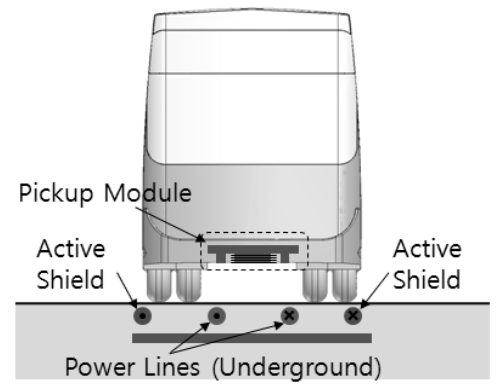

Fig. 13. Concept of active shield for OLEV.

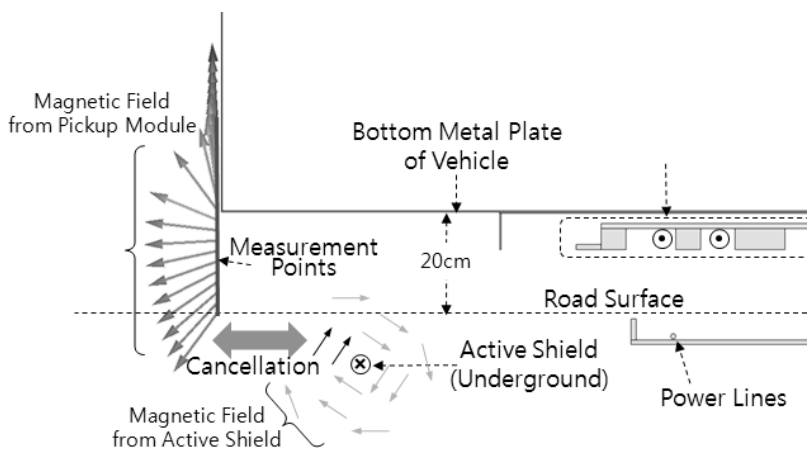

Fig. 14. Direction of magnetic field from pick-up module and active shield.

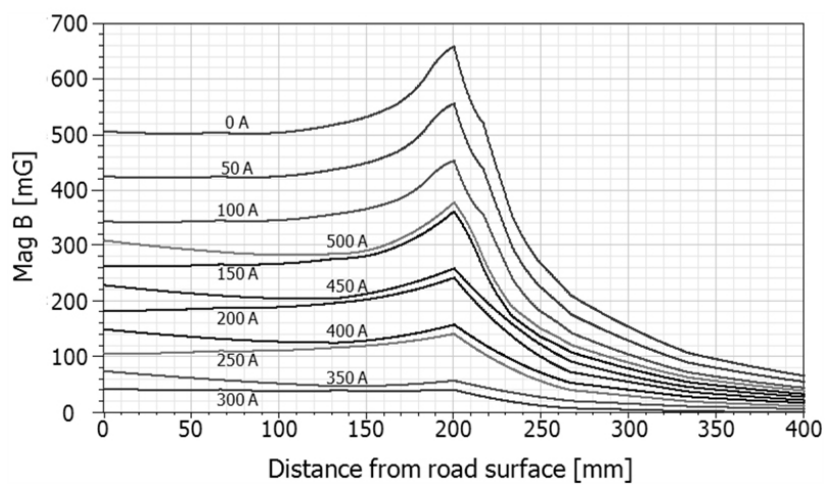

Fig. 15. Change of EMF level according to the current of active shield.

and current magnitude. At the optimal value of current, the magnetic flux density is reduced to $1 / 10$ of the density without the active shield, as depicted in Fig. 15.

\section{Optimization of Design Parameters}

To optimize the design, we first formulate a parameter optimization problem, such that the transferred power to the pick-up, $P_{\text {Transfer }}$ which is consumed at the load $R_{L}$ of Fig. 6, is maximized while EMF level and power transfer efficiency, $K$ satisfy the requirements. We assume that the power transfer efficiency should be greater 
Table 2. System parameters.

\begin{tabular}{|c|c|}
\hline \multicolumn{2}{|c|}{ Constant system parameters } \\
\hline Air-gap & $g_{\text {Air }}(=20 \mathrm{~cm})$ \\
\hline Resonance frequency & $f(=20 \mathrm{kHz})$ \\
\hline Parasitic resistance of power lines & $R_{1}(=0.1 \Omega)$ \\
\hline Parasitic resistance of pick-up coil & $R_{2}(=0.1 \Omega)$ \\
\hline Load resistance & $R_{L}(=10 \Omega)$ \\
\hline System design parameters \\
\hline Width of pick-up coil & $W_{C}$ \\
\hline Current of power lines & $I_{S}$ \\
\hline Number of turns in pick-up coil & $N$ \\
\hline
\end{tabular}

than or equal to 0.8 , and the EMF leakage should be less than or equal to $62.5 \mathrm{mG}$.

Table 2 shows system parameters, which are divided into two categories: constant system parameters and variable system design parameters. We assume that the air-gap between power lines and pick-up coils, resonance frequency, parasitic resistance of power lines, parasitic resistance of pick-up coil, and load resistance are given as in Table 2. We can change three system design parameters: width of pick-up coil $W_{C}$, current of power lines $I_{S}$, and number of turns in pick-up coil $n$.

Accordingly, we formulate our optimization problem as follows:

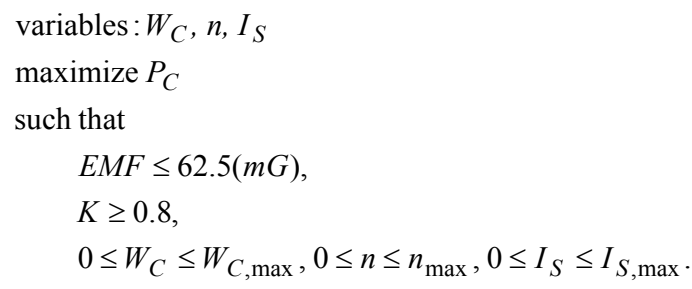

where $W_{C, \max }, n_{\max }$, and $I_{S, \max }$ are the allowable maximum values of $W_{C}, n, I_{S}$, respectively. To solve our problem, we need to express $P_{C}, K, E M F$ in terms of $W_{C}, n, I_{S}$. Since $V_{C}=j(2 \pi f) M n I S$, the induced voltage $V_{C}$ is proportional to $f, n$, and $I_{S}$. Moreover, the EMF is proportional to $n$ and $I_{S}$. Fig. 16 shows the effect of $W_{C}$ on $V_{C}$ and the $E M F$. The difference between the simulation result and the mathematical model should be minimized to improve the accuracy of the design parameter optimization procedure.

From Fig. 16, we obtain the approximate expressions for $V_{C}$ and $E M F$ as follows:

$$
\begin{aligned}
& \left|V_{C}\right| \approx c_{1} f n I_{S} \sqrt{W_{C}}, \\
& E M F \approx c_{2} n I_{S} W_{C}{ }^{2}
\end{aligned}
$$

where $C_{1}$ and $C_{2}$ are constants. Then, transfer power
$P_{\text {Transfer }}$ and total power $P_{\text {Total }}$ at resonant frequency can be represented as:

$$
\begin{aligned}
& P_{\text {Transfer }}=\frac{V_{C}^{2}}{R_{C}} \approx \frac{c_{1}^{2}}{R_{C}} f^{2} n^{2} I_{S}^{2} W_{C}, \\
& P_{\text {Total }} \approx R_{1} I_{S}^{2}+\frac{c_{1}^{2}}{R_{C}} f^{2} n^{2} I_{S}^{2} W_{C} .
\end{aligned}
$$

Therefore, the power transfer efficiency is:

$$
K=\frac{P_{\text {Transfer }}}{P_{\text {Total }}} \approx\left(1+\frac{R_{1} R_{L}}{c_{1}^{2} f^{2} n^{2} W_{C}}\right)^{-1} .
$$

From (5), (6), (8), we can express the optimization problem in (1) as follows:

$$
\begin{aligned}
& \underset{\mathrm{n}, \mathrm{I}_{\mathrm{S}}, W_{C}}{\operatorname{maximize}} \alpha_{1} f^{2} n^{2} I_{S}^{2} W_{C} \\
& \text { such that } \\
& \qquad n I_{S} W_{C}^{2} \leq \alpha_{2} \\
& \qquad f^{2} n^{2} W_{C} \geq \alpha_{3} \\
& \quad 0 \leq W_{C} \leq W_{C, \text { max }}, 0 \leq n \leq n_{\text {max }}, 0 \leq I_{S} \leq I_{S, \text { max }}
\end{aligned}
$$

where

$$
\alpha_{1}=\frac{c_{1}}{R_{C}}, \quad \alpha_{2}=\frac{62.5}{c_{2}}, \quad \alpha_{3}=\frac{R_{1} R_{L}}{c_{1}^{2} f^{2}\left(\frac{1}{0.8}-1\right)} .
$$

Let $x=\log (n), y=\log \left(I_{S}\right), z=\log \left(W_{C}\right)$. Then, the optimization problem in (9) can be restated as:

$$
\begin{aligned}
& \underset{x, y, z}{\operatorname{maximize}} 2 x+2 y+z+\beta_{1} \\
& \text { such that } \\
& \qquad \begin{array}{l}
x+y+2 x \leq \beta_{2} \\
2 x+z \geq \beta_{3} \\
\\
x \leq x_{\max }, y \leq y_{\max }, z \leq z_{\max }
\end{array}
\end{aligned}
$$

where

$$
\begin{aligned}
& \beta_{i}=\log \left(\alpha_{i}\right), i=1,2,3, x_{\max }=\log \left(n_{\max }\right), \\
& y_{\text {max }}=\log \left(I_{S, \text { max }}\right), z_{\text {max }}=\log \left(W_{C, \text { max }}\right) .
\end{aligned}
$$

Note that the problem (10) is a form of typical linear programming (LP) problem.

In the process of finding optimal design parameters, the parameters which maximize transfer power are determined. The width of pick-up coil should be minimized because it increases EMF more significantly than current and number of turns. Similarly, the current and the number of turns should be increased unless it violates the boundary conditions. The boundary conditions on 


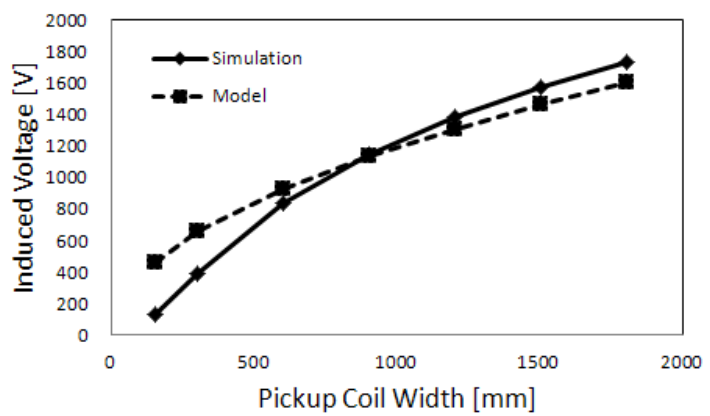

(a) Effect of $W_{C}$ on induced voltage

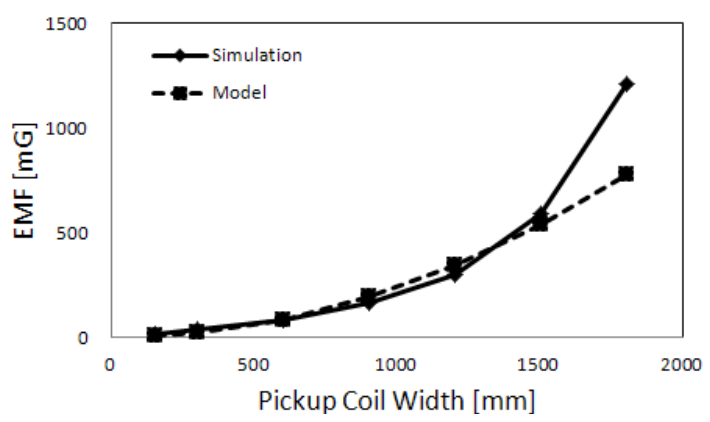

(b) Effect of $W_{C}$ on EMF

Fig. 16. Simulation data and approximation with equation for the effect of $W_{C}$ on induced voltage $V_{C}$ and EMF.

power transfer efficiency affect the design parameters when the frequency is low or mutual inductance is small. Once the product of frequency and mutual inductance is large enough, the EMF is the only boundary condition, and then the combination of the design parameters is determined to make the EMF $62.5 \mathrm{mG}$, which is the maximum value allowed in the optimization. In this EMF boundary, the current and number of turns are maximized until they reach the maximum value we set as $W_{C, \max }, n_{\max }, I_{S, \max }$ in (9). Finally, two maximum values of $n_{\max }, I_{S, \max }$ determine the transferred power because the number of turns and current should reach the maximum value for maximum power.

Now, we obtain the optimal solution for the problem (10) and compare it with the simulation results to investigate the validity of the approximation for LP formulation. Fig. 17 shows the optimal transfer power $P_{C}$ and the variation of constraints such as $E M F$ and $K$ for different values of the frequency $f$. The optimal power increases as the frequency increases because frequency simply increases the transfer power and has no effect on EMF. The efficiency and EMF should be maintained at the specific level. We can find that the simulation results are similar to the LP solution, which means that the approximation for LP formulation is reasonable. More accurate results can be obtained by ap-

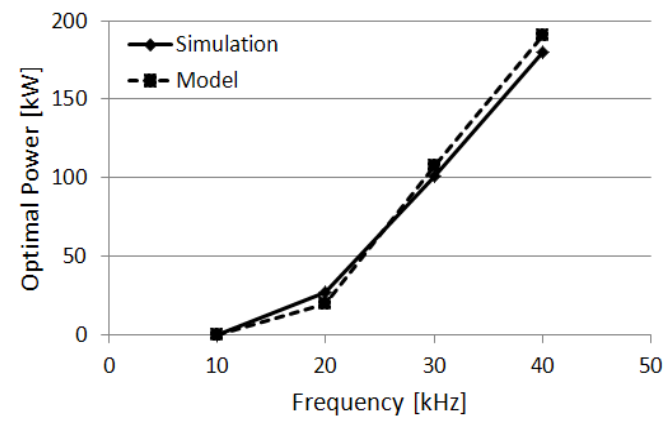

(a) Optimal power

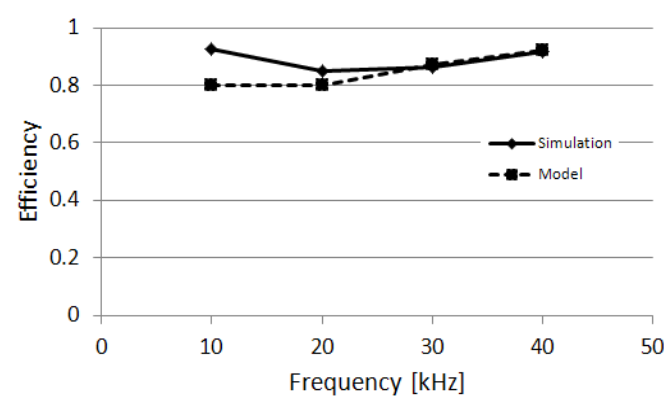

(b) Efficiency, $K$

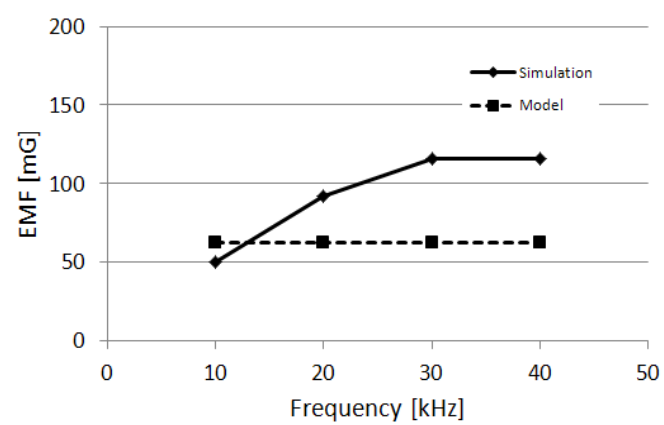

(c) EMF

Fig. 17. Optimal transferred power, efficiency, and EMF for different values of frequency.

plying more complex numerical models in (4) and (5), which describes the voltage and EMF more accurately.

\section{Conclusions}

Design methodologies for the high efficiency of power transfer and the reduction of electromagnetic fields from the system have been proposed. To achieve $80 \%$ of the power transfer efficiency with $60 \mathrm{~kW}$ of power transfer capability, we suggested a vertical magnetic flux type pick-up coil and optimized the design parameters. The design of series resonant coils and frequency selection were the key design factor in securing high OLEV system efficiency. Also, a passive metallic plate shield and active shield are proposed to minimize EMF leakage from the OLEV wireless power transfer system. By 
applying these shielding techniques to a commercial product, we achieved EMF levels lower than $62.5 \mathrm{mG}$.

\section{References}

[1] N. Tesla, "Apparatus for transmission of electrical energy," U.S. Patent 649, 621, May 1900.

[2] A. Kurs, A. Karalis, R. Moffatt, J. D. Joannopoulos, P. Fisher, and M. Soljačić, "Wireless power transfer via strongly coupled magnetic resonances," Science, vol. 317, no. 5834, pp. 83-86, 2007.

[3] S. Kong, M. Kim, K. Koo, S. Ahn, Bumhee Bae, and J. Kim, "Analytical expressions for maximum transferred power in wireless power transfer systems," IEEE International Symposium on Electromagnetic Compatibility, pp. 379-383, 2011.

[4] iSuppli, "Wireless Charging Market Set to Expand by Factor of Nearly 70 by 2014," Jun. 2010.

[5] Cahners In-Stat, "Cut the Cord: Wireless Charging Systems Analysis and Forecast," Aug. 2010.

[6] Systems Control Technology, Inc., "Roadway powered electric vehicle project track construction and testing program phase 3D," California PATH Research Paper, Mar. 1994.

\section{Seungyoung Ahn}

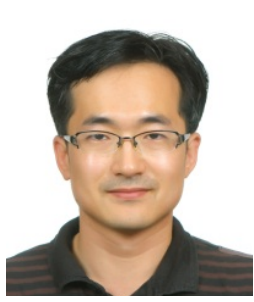

received the B.S., M.S., and Ph.D. degrees in electrical engineering from the Korea Advanced Institute of Science and Technology (KAIST), Daejeon, Korea, in 1998, 2000, and 2005, respectively. $\mathrm{He}$ was with Samsung Electronics as a senior engineer and worked on the design of multi gigabit PCB design for laptop computer system, from 2005 to 2009 . He designed on-line electric vehicle with wireless power transfer technology as a research professor in the department of electrical engineering at KAIST from 2009 to 2011 . He is currently an assistant professor in ChoChunShik Graduate School for Green Transportation at KAIST. His research interest centers on the design of wireless power transfer system in vehicle applications and high speed digital system with consideration of signal integrity and electromagnetic compatibility.
[7] S. Ahn, J. Pak, T. Song, J. Lee, J. Byun, D. Kang, C. Choi, E. Kim, J. Ryu, M. Kim, Y. Cha, Y. Chun, C. Rim, J. Yim, D. Cho, and J. Kim, "Low frequency electromagnetic field reduction techniques for the on-line electric vehicle (OLEV)," IEEE Int'l Symposium on Electromagnetic Compatibility, pp. 625-630, Aug. 2010.

[8] ICNIRP Guidelines, "Guidelines for limiting exposure to time-varing electric, magnetic, and electromagnetic fields (UP TO $300 \mathrm{GHz}$ )," Health Physics, vol. 74, no. 4, Apr. 1998.

[9] L. Hasselgren, J. Luomi, "Geometrical aspects of magnetic shielding at extremely low frequencies," IEEE Trans. on Electromagnetic Compatibility, vol. 37, no. 3, pp. 409-420, Aug. 1995.

[10] W. M. Frix, G. G. Karady, "A circuital approach to estimate the magnetic field reduction of nonferrous metal shields," IEEE Trans. on Electromagnetic Compatibility, vol. 39, no. 1, pp.24-32, Feb. 1997.

[11] C. Buccella, M. Feliziani, and V. Fuina, "ELF magnetic field mitigation by active shielding," IEEE International Symposium on Industrial Electronics, vol. 3, pp. 994-998, Nov. 2002.

Yangbae Chun

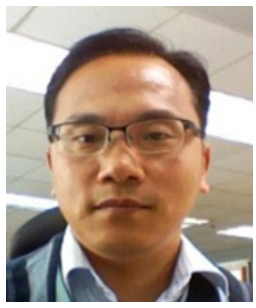

received the B.S. degree in Electronics from Hanyang C. University, Korea in 2004. His interest part is the reduction of unwanted magnetic field on OLEV. Since joining MOTOROLA Korea in 1995, his experience was failure analysis of RF Device, Auto-calibration system programming with Lab-View and programming of Reliability system with HP-Vee. He moved to NOKIA Korea in 2000 and designed several cellular phone models (Leo-plus project) for EMI/EMC and SAR. He moved again to Korea Advanced Institute of Science and Technology (KAIST) OLEV in 2009 and He is currently responsible for the EMC analysis and design of the wireless charging electric vehicle as well system integration. 


\section{Dong-Ho Cho}

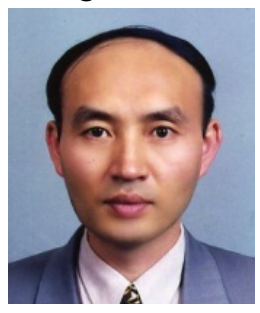

received a B.S. degree in Electrical Engineering from Seoul National University, in 1979 and M.S. and Ph.D. degrees in Electrical and Electronics engineering from the Korea Advanced Institute of Science and Technology (KAIST), in 1981 and 1985, respectively. Between 1987 and 1997, he was a Professor of Computer Engineering at Kyunghee University. Since 1998, he has been a Professor of Electrical Engineering at KAIST. Also, he served as the Advisor officer of Ministry of Information \& Communication (2003-2006), and a Chief of Center for Next Generation Mobile Communication at Ministry of Science and Technology (2004-2006). This center in which he worked as a chief was chosen as the best center among 10 centers for next generation growth engine supported by Korean government for five years. Also, he was selected as a "KAISTIAN of the year" (2007) and received "President Award" from Ministry of Government administration Home Affairs (2006) due to the major contribution to the activation of technology and business for Mobile WiMAX. He has been working as a vice president of KAIST since 2011, a Director of Online Electric Vehicle Project since 2009 and a director of the KAIST Institute for IT convergence since 2007. His research interests include wireless communication, magnetic communication, bio informatics and wireless power transfer. He has published 120 international journal papers and 195 international conference papers.

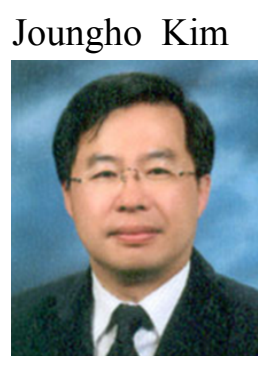

received B.S. and M.S. degrees in electrical engineering from Seoul National University, Seoul, Korea, in 1984 and 1986, respectively, and Ph.D. degree in electrical engineering from the University of Michigan, Ann Arbor, in 1993. In 1994, he joined Memory Division of Samsung Electronics, where he was engaged in Gbit-scale DRAM design. In 1996, he moved to KAIST (Korea Advanced Institute of Science and Technology). He is currently EECS Department Chair at KAIST. Since joining KAIST, his research centers on EMC modeling, design, and measurement methodologies of 3D IC, System-in- Package (SiP), multi-layer PCB, and wireless power transfer technology. Especially, his major research topic is focused on chippackage co-design and simulation for signal integrity, power integrity, ground integrity, timing integrity, and radiated emission of 3D IC and SiP. He has successfully demonstrated low noise and high performance designs of numerous SiP's for wireless communication applications such as ZigBee, T-DMB, NFC, and UWB. Recently, he started a new research on wireless power transfer technology using magnetic field resonance. He has been one of the co-leaders in a national project, OLEV (Online Electrical Vehicle), for EMI and EMF reduction design. The OLEV was selected as one of the 50 Best Inventions in 2010 by Times Magazine. He has authored and co-authored over 330 technical papers published at refereed journals and conference proceedings in modeling, design, and measurement of 3D IC, SiP, PCB, and wireless power transfer. Also, he has given more than 170 invited talks and tutorials at the academia and the related industries. 